\title{
Assessment of the added value of the Twente Photoacoustic Mammoscope in breast cancer diagnosis
}

Marjolein P Hilgerink'

Marjan JM Hummel ${ }^{2}$

Srirang Manohar ${ }^{3}$

Simon R Vaartjes'

Maarten J IJzerman ${ }^{2}$

'Department of Medical Physics, Medisch Spectrum Twente, Enschede, The Netherlands; ${ }^{2}$ Health Technology and Services Research, ${ }^{3}$ Biomedical Photonic Imaging, MIRA Institute, University of Twente, Enschede,

The Netherlands
Correspondence: Marjolein Hilgerink Medisch Spectrum Twente, Algemene Klinische Fysica, Postbus 50.000,

7500 KA Enschede,

The Netherlands

Tel +3I 534873584

Email m.hilgerink@mst.nl
This article was published in the following Dove Press journal:

Medical Devices: Evidence and Research

27 July 201 I

Number of times this article has been viewed

Purpose: Photoacoustic (PA) imaging is a recently developed breast cancer imaging technique. In order to enhance successful clinical implementation, we quantified the potential clinical value of different scenarios incorporating PA imaging by means of multi-criteria analysis. From this analysis, the most promising area of application for PA imaging in breast cancer diagnosis is determined, and recommendations are provided to optimize the design of PA imaging.

Methods: The added value of PA imaging was assessed in two areas of application in the diagnostic track. These areas include PA imaging as an alternative to x-ray mammography and ultrasonography in early stage diagnosis, and PA imaging as an alternative to Magnetic Resonance Imaging (MRI) in later stage diagnosis. The added value of PA imaging was assessed with respect to four main criteria (costs, diagnostic performance, patient comfort and risks). An expert panel composed of medical, technical and management experts was asked to assess the relative importance of the criteria in comparing the alternative diagnostic devices. The judgments of the experts were quantified based on the validated pairwise comparison technique of the Analytic Hierarchy Process, a technique for multi-criteria analysis. Sensitivity analysis was applied to account for the uncertainty of the outcomes.

Results: Among the considered alternatives, PA imaging is the preferred technique due to its non-invasiveness, low cost and low risks. However, the experts do not expect large differences in diagnostic performance. The outcomes suggest that design changes to improve the diagnostic performance of PA imaging should focus on the quality of the reconstruction algorithm, detector sensitivity, detector bandwidth and the number of wavelengths used.

Conclusion: The AHP method was useful in recommending the most promising area of application in the diagnostic track for which PA imaging can be implemented, this being early diagnosis, as a substitute for the combined use of x-ray mammography and ultrasonography.

Keywords: technology assessment, breast cancer, diagnostic imaging, biomedical engineering

\section{Introduction}

At the current rates of incidence, about 1 in 8 women in the Netherlands will develop breast cancer, and 1 in 22 women will die of the disease. ${ }^{1}$ Incidence risk increases in the middle years of a woman's life rather than the advanced years as with other types of cancer. Thus, the number of years lost by those succumbing to breast cancer is higher than with other cancers. The current paradigm of breast cancer mortality reduction is based on early detection followed by effective intervention.

The diagnosis of breast cancer in Europe is based on triple assessment: clinical examination; imaging; and cytological/histological sampling, often in a single visit. The accuracy of triple assessment depends on the quality of each constituent test. ${ }^{2,3}$ 
The most frequently used imaging techniques within the triple assessment are x-ray mammography and ultrasonography, either alone or in combination. Magnetic resonance imaging (MRI) is also becoming more established and widely used. ${ }^{3}$

There are several drawbacks regarding the use of these imaging techniques. X-ray mammography offers poor contrast in radiodense glandular breasts, and the use of ionizing radiation offers some concern due to its associated carcinogenic potential. Furthermore, patients complain about the discomfort in x-ray mammography. Ultrasonography plays an adjunctive role to $\mathrm{x}$-ray imaging in solid mass-cyst differentiation. However, there is not yet any large-scale application of the technique to differentiate malignant from benign abnormalities, and the results are strongly operator dependent. MRI is gaining a niche role as a problem solving technique in certain cases such as with uncertain findings in mammography and ultrasonography. However, it requires the use of contrast agent, and has shown poor specificity. Moreover, MRI is more expensive than the other imaging techniques, and therefore has limited accessibility. ${ }^{4}$ In conclusion, all these techniques have several drawbacks felt both at individual and societal levels: distress, pain, false reassurance, morbidity and mortality, as well as direct and indirect financial burden.

A new technology, photoacoustic (PA) imaging, has recently gained much attention in imaging various pathological states related to vascular condition and function. ${ }^{5}$ Some important applications of this technique include breast cancer visualization. ${ }^{6,7}$ This technique has the potential to address at least some of the drawbacks of the conventional techniques. The technique relies on irradiating tissue with nanosecond pulses of visible or near infrared (NIR) light. Optical absorption in tissue causes thermoelastic expansion, which produces broadband pulses $(\mathrm{MHz})$ of ultrasound energy. These pulses propagate through the tissue and can be detected at the tissue surface at multiple positions using ultrasound detectors. ${ }^{7}$ Tumor growth is associated with enhanced blood vessel supply generated in the process called angiogenesis. Blood in this enhanced vascularization absorbs light strongly and endows an absorption contrast to the cancer. Therefore, the PA imaging technique might be valuable for the large group of women potentially suffering from breast cancer. The proof of principle of PA imaging in breast cancer detection has been demonstrated in restricted studies using near-infrared (NIR) light in an instrument developed at the University of Twente (the Netherlands), called the Twente Photoacoustic Mammoscope PAM. ${ }^{7,8}$
As the choice for any imaging technique involves multiple decision criteria, a formal assessment of the added value of a new diagnostic device should take a prioritized set of criteria into account. For the implementation of PA imaging, different areas of application in the diagnostic track for breast cancer can be chosen, each with their own benefits and drawbacks, and relevant criteria. Early technology assessment can be applied to evaluate the possible success of PA imaging in each of these areas of application. Forward thinking about the future clinical application of a technology that is still in development is also part of Constructive Technology Assessment (CTA) and Real-time Technology Assessment (RTA). Such early assessments of a technology that take into account expert judgment and discussion, early data on patients, and first experiences with a technology, can result in relevant recommendations. These recommendations can fine-tune the further development of the new technology, such as PA imaging, to the requirements of clinical practice. This has also been demonstrated in different studies using CTA or RTA for early assessment of nanotechnology and oncology applications. ${ }^{9-17}$ Early technology assessment can be conducted by using multi-criteria decision analysis (MCDA) methods. Multi-criteria decision analysis can support the evaluation of a technology with respect to different outcome measures. It helps decision-makers to evaluate a finite number of alternatives under a finite number of performance criteria. Saaty's mathematical model, the analytic hierarchy process (AHP), is one of these techniques for multi-criteria decision analysis. ${ }^{18}$ It was initially introduced to support strategic decisions in industry, but the technique is also applicable in different stages of health technology development and diffusion. $^{19}$

In this study, we applied AHP analysis to an initial comparison of the expected performance of PA imaging with conventional diagnostic mammography techniques. This systematic, transparent method of assessment by a small, multidisciplinary group of experts will provide the developers of PA imaging with useful feedback about possible improvements to their technique, and can aid decisions they still need to make. We used the outcomes of the analysis to determine the most promising area of application for PA imaging in breast cancer diagnosis.

\section{Methods \\ Defining areas of application in the diagnostic track for breast cancer}

Diagnosis of breast cancer mainly starts with clinical examination, followed by basic imaging procedures, such as 
mammography and ultrasonography, prior to any clinically guided tissue sampling. The use of these basic imaging procedures will be referred to as "early diagnosis" in this study. Mostly, early diagnosis starts with mammography. Ultrasound imaging is used if the tumor is mammographically occult, but also if visible on the x-ray as part of the standard triple assessment procedures. ${ }^{2,3}$ Further ultrasonography is the initial diagnostic imaging method applied for women under the age of 35 years, where the use of x-rays is not recommended. ${ }^{2,3}$

MRI is increasingly used in later stages of breast cancer diagnosis. MRI is of proven value in helping to establish the degree of disease present where malignancy is already established or highly likely in dense breasts, or with tumors having a likelihood of multifocality, multicentricity or bilaterality. MRI has also been shown to have a high sensitivity in the detection of malignancy in younger women at high risk, and MRI is currently recognized as the method of choice for investigating significant abnormalities in the breast in the presence of implants. The use of MRI in breast cancer diagnosis will be referred to as "late diagnosis" in this study. ${ }^{3}$

Both early and late diagnosis are expected to be suitable areas of application for PA imaging in breast cancer diagnosis.

\section{AHP analysis}

The first step in the AHP analysis is to define the framework, or hierarchical structure, for the analysis. This framework consists of the goal, criteria and the alternative imaging techniques.

\section{Goal}

The goal of this AHP analysis is to compare the expected performance of PA imaging with the conventional techniques used in different areas of application in the diagnostic track for breast cancer.

\section{Criteria}

To identify relevant criteria for AHP analysis, a literature search was performed, followed by several discussions at the Centre for Mammacare in a large Dutch teaching hospital. On the basis of the literature study and discussions with engineers and radiologists, relevant factors were identified that determine the quality of a breast imaging device. These factors were used to compose the hierarchical evaluation structure (see Figure 1).

The top-level criteria in the hierarchical structure: costs; effectiveness; patient comfort; and risks, are from the European guidelines for breast cancer diagnosis. ${ }^{3}$ The costs subcriteria followed from an interview with the finance department of the Dutch teaching hospital. The effectiveness subcriteria were identified from clinical breast imaging guidelines and literature, and from the world-wide classification standard for breast lesions, BI-RADS.,20 The most frequently mentioned tumor aspects in these documents are presented in the hierarchy. Oxygen saturation was added to the effectiveness subcriteria, because the presentation of information about oxygen saturation of the blood associated with the tumor is a unique feature that PA imaging potentially offers, and the AHP results may show if this new feature will be important. Patient comfort subcriteria were derived

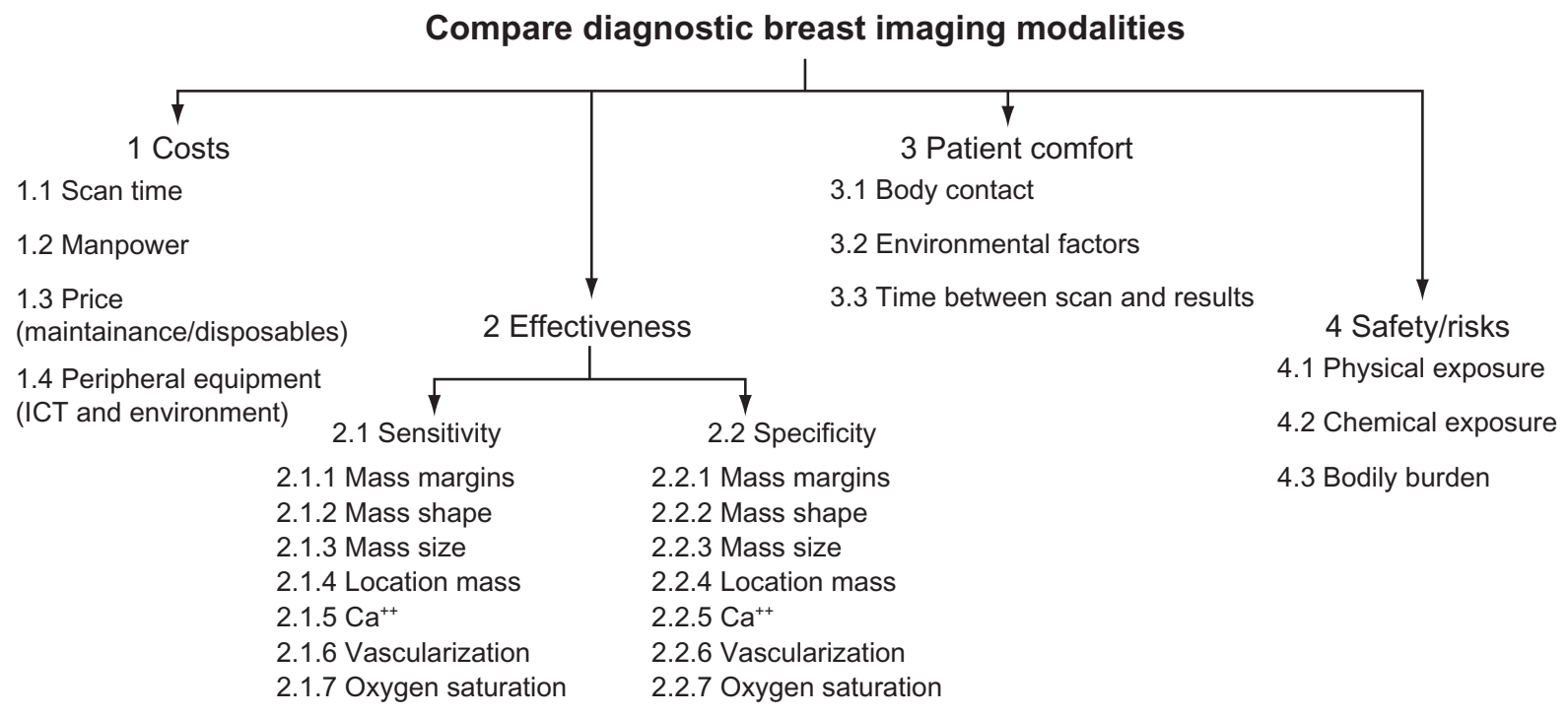

Figure I AHP hierarchical structure. Abbreviation: AHP, analytic hierarchy process. 
from patient information from different cancer institutes, societies, and patient organizations. ${ }^{21}$ The risks subcriteria were determined from incident analyses and an interview with a medical physicist.

\section{Alternatives}

PA imaging was compared to conventional mammography techniques used in both early and late diagnosis.

Only a limited amount of data and clinical prototypes are available for PA breast imaging. ${ }^{7,8}$ We thus used the technical specifications and performance (past and predicted) of the Twente Photoacoustic Mammoscope, PAM II, as being representative for PA imaging in general. Comparisons are made with the current technologies that PA imaging aims to substitute or will compete with: x-ray mammography, ultrasonography and MRI.

PAM II is a prototype PA imaging device being developed by the Biomedical Photonic Imaging group of the University of Twente. An overview of the expected specifications of PAM II is provided in Table 1. PAM II will produce tomographic images of the full breast. During scanning, the patient lies in a prone position with her breast hanging in an imaging tank filled with water at body temperature. The laser and detector matrix spin around in this tank and multiple projections of the breast are acquired. The use of a dedicated reconstruction algorithm and multiple wavelengths of light in this prototype will offer the possibility of the production of images of vascularization associated with breast carcinoma, and information about the level of oxygen saturation of the blood in the tumor vessels.

Table I Overview of expected specifications PAM II

\begin{tabular}{ll}
\hline Item & Value \\
\hline Scan time & $15-20$ minutes per breast \\
Expected price of purchase & $€ 400.000$ \\
In plane resolution & $<\mathrm{I} \mathrm{mm}$ \\
Depth resolution & $<\mathrm{I} \mathrm{mm}$ \\
Max. imaging depth & $50+\mathrm{mm}$ \\
Reconstruction & $3 \mathrm{D}$ \\
Scan area & Full-breast (40 mm radius) \\
Patient position & Prone position \\
Breast compression & None \\
Radiation hazard & Non-ionizing radiation \\
& Laser safety measures needed \\
Availability of results & Within 2-3 days, after evaluation \\
& by trained radiologist \\
Contrast medium & Blood \\
Functional tumor aspects visible & No contrast agent needed \\
& Blood vessels \\
& Oxygen saturation (\%) \\
\hline
\end{tabular}

Table 2 Composition of AHP expert team

\begin{tabular}{|c|c|c|}
\hline No. & Profession & Core PAM activity/relation \\
\hline 1 & Manager* & Costs for health care organization \\
\hline 2 & $\begin{array}{l}\text { Medical specialist } \\
\text { (Radiologist)* }\end{array}$ & User \\
\hline 3 & $\begin{array}{l}\text { Nurse practitioner/ } \\
\text { radiology assistant* }\end{array}$ & User, patient representative \\
\hline 4 & Physicist & Technical design of PAM \\
\hline 5 & Laser physicist & $\begin{array}{l}\text { Technical design of PAM, } \\
\text { optical engineering }\end{array}$ \\
\hline 6 & Physicist & $\begin{array}{l}\text { Research in one of the world's } \\
\text { leading medical device companies, } \\
\text { main interest in optical mammography }\end{array}$ \\
\hline 7 & Medical physicist & Safety and Quality assurance \\
\hline
\end{tabular}

It is assumed that PA imaging will replace one or more conventional techniques, instead of being used as an additional technique in the diagnostic track. This assumption is made because additional methods usually result in an extended diagnostic pathway at higher cost and therefore prevent implementation. This means that, for early diagnosis, the alternatives used in the AHP analysis are: the combined use of $x$-ray mammography and ultrasonography; the combined use of $\mathrm{x}$-ray mammography and PAM II (PAM II as a substitute solely for ultrasonography); and the use solely of PAM II (PAM II as a substitute for both x-ray mammography and ultrasonography). For late diagnosis, the alternatives used in the AHP analysis are: MRI and PAM II. Both sets of alternatives, each corresponding to one area of application, were evaluated separately, because they are implemented for other patient groups, and other performance data may be important.

\section{Expert panels}

Two expert teams were invited to participate in the AHP analysis, with the inclusion of appropriate professional experts in case of early diagnosis and late diagnosis. In interviews with the developers of PAM II and with different medical experts, a multidisciplinary panel of experts was identified. Table 2 presents the invited team members together with their professional backgrounds.

\section{AHP session}

All members of the AHP expert team had received information about the AHP hierarchy and a performance matrix, containing relevant facts about all alternatives, in advance. A day-section feedback session was organized in which the process. 
expert team discussed the relative importance of the criteria, subcriteria, and the pursued quality of each diagnostic alternative. This was supported using Team Expert Choice software (Expert Choice, Arlington, VA), a commercially available group decision support system that incorporates the mathematical procedures of the AHP. ${ }^{22}$ The feedback session started with an introduction of the software and the procedures of Team Expert Choice and the developer of PAM II explained the principles of this diagnostic device. The AHP hierarchy was also explained. Then, using handheld remote controlled keypads, the members of the expert team provided their judgments on pairwise comparisons of the importance of the selected subcriteria, and preference for the selected alternatives. Individual judgments were projected on a screen, allowing the members of the expert team to discuss the rationales behind their individual scores. During the discussions, the expert team members had the opportunity to alter their judgments. To support the team members and to make sure every expert used the same definition of a subcriterion, the descriptions of the subcriteria that were assessed were displayed on a second screen. For each pairwise comparison, the final individual judgments were aggregated, based on the geometric mean, to create group weighting factors representing the importance of the subcriteria and the priorities reflecting the relative preference for the alternatives.

\section{Sensitivity analysis}

Because PA imaging, and especially PAM II, is early in its development and only a limited amount of data has been obtained to date, its performance is uncertain. It is expected that this uncertainty mainly exists regarding the sensitivity and specificity of PAM II. The experts individually assessed this uncertainty about the different pairwise judgments regarding sensitivity and specificity, using a 3-point scale. Based on the uncertainty expressed, three scenarios were constructed: negative, average and positive, for PAM II. These scenarios were used in a sensitivity analysis regarding the predicted performance of PA imaging.

\section{Results}

The results of the AHP analysis are presented in Figure 2, which shows the results of the comparison of PAM II with x-ray mammography and ultrasonography (early diagnosis), and in Figure 3, which shows the results of the comparison of PAM II with MRI (late diagnosis).

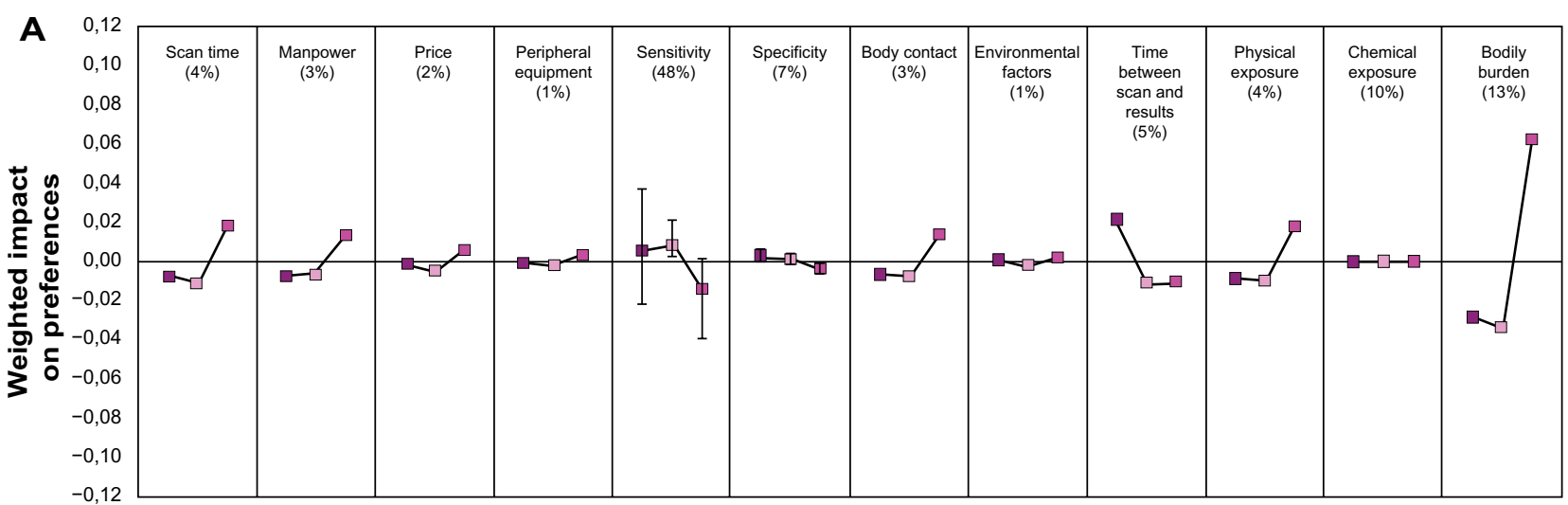

B

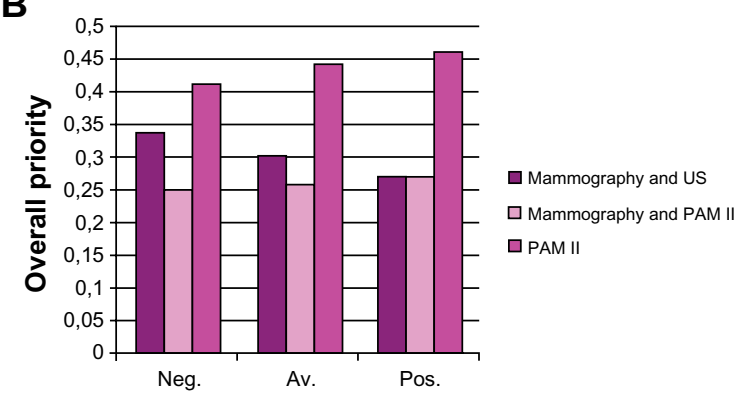

Figure 2 Results of early diagnosis. (A) Importance of the criteria, and relative preference for the breast imaging alternatives with respect to the criteria, early diagnosis. (B) Overall preferences for the alternatives, early diagnosis.

Abbreviations: Neg, Negative scenario; av, average scenario; pos, positive scenario. 

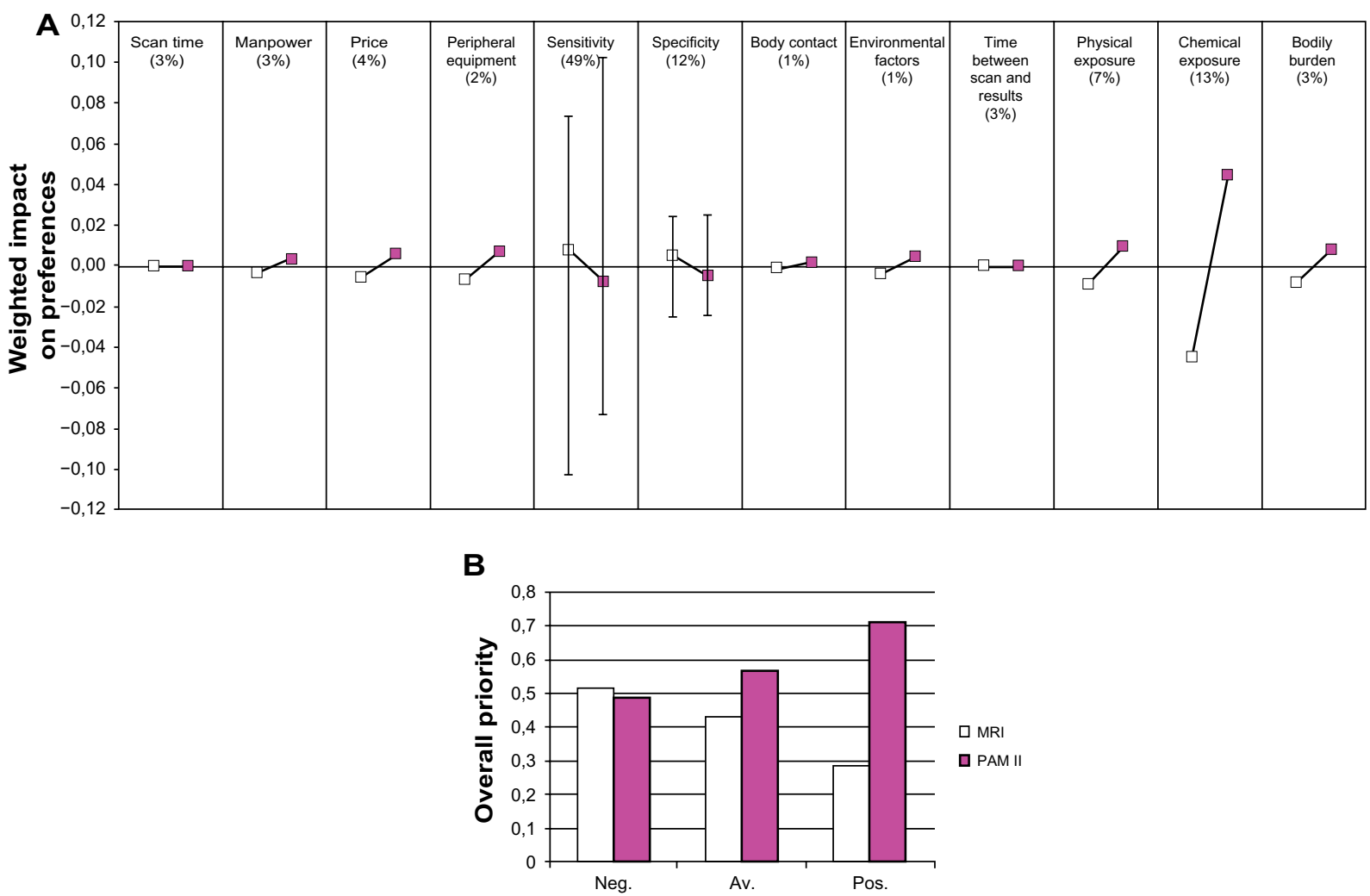

Figure 3 Results of late diagnosis. (A) Importance of the criteria, and relative preference for the breast imaging alternatives with respect to the criteria, late diagnosis. (B) Overall preferences for the alternatives, late diagnosis.

Abbreviations: Neg, Negative scenario; av, average scenario; pos, positive scenario.

At the top of Figures $2 \mathrm{~A}$ and $3 \mathrm{~A}$, the relative importance of the criteria, resulting from the pairwise comparisons in the AHP, is expressed in percentages, between brackets. The lines below each criterion reflect the relative preference for each breast imaging alternative with respect to that criterion, multiplied by the relative importance of that criterion. Thus, a larger deviation from 0 reflects a more discriminating criterion. For example, in the comparison of mammography, ultrasonography and PAM II (Figure 2A), the preference for PAM II with respect to bodily burden is an important contributor to the overall preference for one of the breast imaging alternatives.

The most important aspect of a diagnostic breast imaging device is its diagnostic performance, especially with respect to sensitivity ( $48 \%-49 \%$, see Figures $2 \mathrm{~A}-3 \mathrm{~A})$. This means that a change in the design of a breast imaging device which affects the sensitivity is expected to have the largest impact on the relative preference for that device. However, the performance of the different breast imaging alternatives with respect to sensitivity is not expected to be very different, so "sensitivity" is in this case not the most discriminating factor for the overall preference.

The main factors that determine sensitivity in an image are the representation of mass margins (relative weight $=0.119$ ), mass shape (0.106), vascularization (0.106) and oxygen saturation (0.123). The relative weights for these factors result from the AHP analysis of the subcriteria.

The bars around the average sensitivity and specificity scores in Figures 2A and 3A show the results of the sensitivity analysis applied to the diagnostic performance of PAM II.

In Figures 2B and 3B, the height of the bars reflects the relative preference for each of the alternatives. Three different scenarios resulting from the sensitivity analysis are shown. Figures 2B and 3B show that with increasing positivity, PAM II becomes a more preferred option. Compared to x-ray mammography and ultrasonography, PAM II is, even in the most negative scenario, the most preferred option. This is not true for the performance of PAM II compared to MRI, which shows a slight preference for MRI in the most negative scenario in the sensitivity analysis.

From the figures, it becomes clear that PA imaging is a preferred technique due to its non-invasiveness, low cost and low risk. The experts do however not expect a large difference in the overall diagnostic performance of PA imaging, either with mammography and ultrasonography or with MRI.

Sensitivity was rated as the most important aspect of a diagnostic breast imaging device. The representation of mass 
margins, mass shape, vascularization and oxygen saturation in an image are factors that mainly determine the expected performance with respect to sensitivity. Therefore, in order to improve the diagnostic performance of PAM II, technical improvements should be carried out that are focused on improvement of these factors. These technical improvements will include adjustments to the reconstruction algorithm, the sensitivity of the detector, the bandwidth of the detector, and the number of wavelengths of light used.

\section{Discussion and conclusion}

The goal of this study was to compare PA imaging with conventional diagnostic mammography techniques, in order to determine the most promising area of application for PA imaging in breast cancer diagnosis. The overall results indicated that, based on the expected performance in terms of cost, effectiveness, patient comfort and risk criteria assessed, PAM II will be a preferred alternative. At the current stage, the experts' judgments do not result in strong differences in the overall value of the alternative diagnostic devices. The sensitivity analysis showed that only in the most negative scenario, will MRI be a preferred alternative to PAM II. All other scenarios result in PAM II being the most preferred option.

The most promising area of application for PA imaging is expected to be early diagnosis, as a substitute for the combined use of x-ray mammography and ultrasonography. This alternative was preferred even in the negative scenario in the sensitivity analysis (Figures 2B-3B). In addition, the preference for PAM II with respect to the risk and cost factors was larger when PAM II was compared to x-ray mammography and ultrasonography, than when PAM II was compared to MRI.

The results showed that PAM II was preferred over the other alternatives with respect to physical exposure (Figure 2A-3A), chemical exposure (Figure 3A) and bodily burden (Figure 2A-3A). Because PAM II does not make use of potentially harmful ionizing radiation, physical exposure is less than for both the mammography options. Furthermore, in the conventionally used x-ray mammography devices, the breast is forced between two plates, which can be a painful experience. Because in PAM II the breast is situated in an imaging tank without any compression, PAM II is a strongly preferred option regarding bodily burden. Compared to MRI, the non-invasiveness of the PAM II procedure was preferred over the invasive procedure of MRI, which requires the injection of contrast agent. This contrast agent is also expected to carry a small risk regarding chemical exposure.
Furthermore, costs are expected to be lower for PAM II, mainly because only the use of a single device is needed opposite to the use of more devices, or the use of an expensive MRI system, in the other alternatives.

In both early and late diagnosis, sensitivity is by far the most important aspect. From the AHP analysis of the sensitivity subcriteria, it followed that the representation of mass margins, mass shape, vascularization, and oxygen saturation in an image are most needed for accurate sensitivity. Investing in the development of a good reconstruction algorithm, a high sensitivity detector, a large detector bandwidth, and a multi-wavelength light source for PAM II, will improve its performance with respect to these criteria.

The AHP proved to be suitable for early technology assessment of PA imaging. Important design criteria were identified, and a substantiated prognosis of the most promising area of positioning PA imaging was given. When applying these methods, the following methodological considerations should be kept in mind.

In our problem definition we made the assumption that PA imaging will be a substitute, therefore we only identified conventional imaging techniques as being alternatives for PA imaging. Looking to the future, there might be other alternatives for PA imaging, such as positron emission tomography - computed tomography (PET-CT) and magnetic resonance elastography (MR-elastography) applications that have not been taken into account in the analysis. These new techniques might be used in addition to the conventional techniques, but their performance and future implementation in health care is unsure. In order to reduce complexity, we only considered imaging techniques applied in clinical practice. However, one needs to keep in mind that other relevant alternatives can arise over time.

The medical, social, industrial and technical backgrounds of the panel members influence their judgments about the importance of the decision criteria and the value of the diagnostic devices. Therefore it is essential that a representative group of experts are asked to participate in an expert panel. In this case, the categories of experts were chosen to match the types of criteria that were assessed. The patient comfort expert was chosen to be a nurse practitioner, as being best able to judge or predict patient response, in the absence of known patients who had experience with PA imaging. The experts were identified based on interviews with the developers of PAM II and with different medical experts. All experts are well-known at national level, for their research and experience with mammographic techniques. We were aware of the fact that the subjectivity of a small group 
could influence the outcomes. However, because insiders with relevant knowledge were represented in our expert team, we obtained relevant outcomes for this early phase of development of PA imaging. In later developmental phases, closer to implementation, it becomes more important to include larger groups of experts, including patients.

Ideally, the experts would have been equally distributed over the different fields, but, in our analysis, the number of engineers was rather large compared to the other experts. To check if the engineers assessed the criteria differently from the other experts, a subgroup analysis was carried out. Results of this subgroup analysis showed that for the engineers, costs and effectiveness were more important than for the other experts. The other experts judged patient comfort and safety as more important aspects than the engineers. However, the relative order of importance of the criteria was similar for both groups. PAM II is slightly less preferred by the engineers than by the other experts, because of the lower expected effectiveness. The experts who were involved in the creation of PAM II did not show deviating judgments from the other engineers.

It was also important that the team had an equal basic knowledge about the new technology, so that discussions focus efficiently on knowledge-sharing between the experts to gain new insights for further development of PAM II. We sent panel members state-of-the-art literature before the session, and staged presentations by the relevant stakeholders before the panel discussions. During the panel session itself we allowed sufficient time for sharing knowledge about the relevant values of the diagnostic devices.

The criteria used in this analysis were identified mainly on the basis of literature and interviews with different experts. We were aware that this set of criteria might not provide a complete view of the situation; the AHP structure becomes too extensive if all relevant criteria have to be taken into account. We chose to define the set of criteria before the AHP session took place. Hummel et al indicated that it is also possible to let the definition of the criteria be part of the AHP session. ${ }^{9}$ This may create more consensus about the most important criteria.

In addition, a consensus still needs to be reached about the number of decision criteria to include in the assessment. More decision criteria increases the amount of detailed information discussed, but increases the cognitive load due to the increased number of pairwise comparisons. The cognitive load of our extensive AHP structure appeared to cause some cognitive fatigue. It is therefore important to keep the hierarchy as simple as possible, and to have a well-informed discussion leader for the AHP, who can keep the expert team focused on the exact content of the issues being addressed.

The interdependence of decision criteria that are used for the AHP analysis should be minimized. Considering the overlap that appeared between some of the subcriteria of costs, we advise using "costs" only as a main criterion in the AHP analysis, and using conventional accountancy methods to calculate the overall costs created by different sub-components.

Taking into account the above aspects, it can be concluded that AHP is able to provide a useful prediction of the possible future success of a new technology. In this case the developers of PA imaging devices know now that their devices have a realistic chance of success, which is an important argument to encourage investment in the further development of the device. During the AHP analysis in this study, useful interdisciplinary discussions took place that provided the developers not only with new insights about the likely values and relevant improvements of PAM II, but also with an opportunity to inform, interact with, and possibly win over a group of potential users, which is expected to enhance acceptability. In addition it provided an overview of the main uncertainties in the outcomes. One example is the presentation of oxygen saturation. There are questions about the exact diagnostic value PAM II can offer with this information. More research is needed about the exact way in which this parameter can support diagnosis. The radiologists were curious about the visualization of this parameter in the image, it is therefore suggested that they judge several concepts, and develop the visualization in cooperation. The outcomes of this assessment indicate relevant directions for further research in diagnostic imaging.

The AHP method proved to be valuable in assessing the value of PA imaging in the diagnostic track for breast cancer. It showed that PA imaging can best be positioned at the start of the diagnostic track as an alternative to $\mathrm{x}$-ray mammography and ultrasonography. Therefore, in the further development of PAM II, more specific issues and questions that arise in early diagnosis should be examined, in order to make PAM II optimally suitable for this diagnostic demand.

Our future research will focus on the use of the AHP method for early technology assessment to assess the value of PA imaging in screening. Application of PA imaging in screening might be beneficial for a lot more women. Current screening methods do not provide completely satisfactory results with respect to effectiveness, and the radiation risk 
from conventional x-ray mammography is still an issue. Also, patient comfort is expected to be more important because a high quality screening program demands good cooperation from the patients. The performance of PA imaging with respect to other alternatives could also be investigated. Nuclear medicine is becoming more popular within cancer diagnosis, and new applications of MRI are being developed, such as MR elastography, that may become serious competitors to PA imaging.

\section{Acknowledgments}

$\mathrm{SM}$ is supported by Agentschap NL Innovation Oriented Research Programmes Photonic Devices in the HYMPACT Project (IPD083374).

\section{Disclosure}

SM has a financial interest in PA Imaging Holding BV. PA Imaging Holding BV did not financially support the research or the publication of this work.

\section{References}

1. Visser O, van Noord KJ, eds. Feiten + Fabels over kanker in Nederland. 1 st ed. Utrecht, the Netherlands: Vereniging van Integrale Kankercentra; 2005.

2. National Institute for Clinical Exellence. Guidance on Cancer Services: Improving Outcomes in Breast Cancer. 1st ed. London, England: NHS; 2002.

3. Perry N, Broeders M, de Wolf C, Törnberg S, Holland R, von Karsa L. European guidelines for quality assurance in breast cancer screening and diagnosis. 4th ed. European Communities: Health and Consumer Protection Directorate-General; 2006.

4. Berg WA, Birdwell RL, Gombos EC, et al. Diagnostic Imaging: Breast. Salt Lake City, Utah: Amirsys Inc; 2008.

5. Wang LV. Multiscale photoacoustic microscopy and computed tomography. Nat Photonics. 2009;3(9):503-509.

6. Ermilov SA, Khamapirad T, Conjusteau A, et al. Laser optoacoustic imaging system for detection of breast cancer. J Biomed Opt. 2009;14(2): 024007.

7. Manohar S, Vaartjes SE, van Hespen JCG, et al. Initial results of in vivo non-invasive cancer imaging in the human breast using near-infrared photoacoustics. Opt Express. 2007;15(19):12277-12285.
8. Piras D, Wenfeng X, Steenbergen W, van Leeuwen TG, Manohar SG. Photoacoustic imaging of the breast using the Twente Photoacoustic Mammoscope: present status and future perspectives. Selected topics in quantum electronics, IEEE journal. 2010;16(4):730-739.

9. Hummel JM. Supporting medical technology development with the analytic hierarchy process. Groningen, the Netherlands: Rijksuniversiteit Groningen, Faculty of Medical Sciences; Dissertation, 2001.

10. Pietzsch JB, Paté-Cornell ME. Early technology assessment of new medical devices. Int J Technol Assess Health Care. 2008;24(1): 36-44.

11. Cosh E, Girling A, Lilford R, McAteer H, Young T. Investing in new medical technologies: a decision framework. J Commercial Biotechnology. 2007;13(4):263-271.

12. Douw K, Vondeling H. Selection of new health technologies for assessment aimed at informing decision making: a survey among horizon scanning systems. Int J Technol Assess Health Care. 2006; 22(2):177-183.

13. Guston DH, Sarewitz D. Real-time technology assessment. Technology in Society. 2002;24(1):93-109.

14. Douma KFL, Karsenberg K, Hummel JM, Bueno-de-Mesquita JM, van Harten WH. Methodology of constructive technology assessment in health care. Int J Technol Assess Health Care. 2007;23(2):162-168.

15. Retèl VP, Hummel JM, van Harten WH. Early phase Technology Assessment of nanotechnology in oncology. Tumori. 2008;94(2): 284-290.

16. Retèl VP, Bueno-de-Mesquita JM, Hummel JM, et al. Constructive Technology Assessment (CTA) as a tool in Coverage with Evidence Development: the case of the 70-gene prognosis signature for breast cancer diagnostics. Int J Technol Assess Health Care. 2009;25(1): 73-83.

17. Rip A. Folk theories of nanotechnologists, Science as Culture, 2006; 15(4):349-365.

18. Saaty TL. The Analytic Hierarchy Process: Planning, Priority Setting, Resource Allocation. New York: McGraw-Hill; 1980.

19. Hummel JM, van Rossum W, Verkerke GJ, Rakhorst G. Assessing medical technologies in development. A new paradigm of medical technology assessment. Int J Technol Assess Health Care. 2000;16(4): 1214-1219.

20. Breast Imaging Reporting and Data System Atlas. BI-RADS $S^{\circledR}$ Atlas. Reston: American College of Radiology (ACR); 2003.

21. Borstkanker. [homepage on the Internet]. The Netherlands: KWF Kankerbestrijding. [updated 2008 Aug 22; cited 2009 May 7]. Available from: http://www.kwfkankerbestrijding.nl/. Accessed May 7, 2009.

22. Hummel JM, van Rossum W, Verkerke GJ, Rakhorst G. The effects of team expert choice on group decision-making in collaborative new product development: a pilot study. Journal of Multi-Criteria Decision Analysis. 2000;9(1-3):90-98.
Medical Devices: Evidence and Research

\section{Publish your work in this journal}

Medical Devices: Evidence and Research is an international, peerreviewed, open access journal that focuses on the evidence, technology, research, and expert opinion supporting the use and application of medical devices in the diagnosis, treatment and management of clinical conditions and physiological processes. The identification of novel

\section{Dovepress}

devices and optimal use of existing devices which will lead to improved clinical outcomes and more effective patient management and safety is a key feature. The manuscript management system is completely online and includes a quick and fair peer-review system. Visit http://www. dovepress.com/testimonials.php to read real quotes from authors. 